\title{
Effects of therapeutic exercise on masticatory function in patients with progressive muscular dystrophy
}

\author{
Y KAWAZOE, M KOBAYASHI, T TASAKA, M TAMAMOTO \\ From the Department of Prosthetic Dentistry, Hiroshima University School of Dentistry, Hiroshima, Japan
}

SUMMARY The slope of the curve relating integrated electromyographic activity of masseter muscle to biting force, the latency of the jaw-jerk reflex, and masticatory performance were estimated in patients with Duchenne type of progressive muscular dystrophy before and during therapeutic exercise of the somatognathic system. The slope and latency were slightly decreased, and masticatory performance was increased during exercise. These results suggest that therapeutic exercise of the stomatognathic system is effective in improving masticatory function in patients with progressive muscular dystrophy.

Previous electromyographic study ${ }^{1}$ analysing masticatory function in patients with the Duchenne type of progressive muscular dystrophy (PMD) has shown that the slope of the curve relating integrated electromyographic activity of the masseter muscles to biting force during rapid isometric contraction of the jaw (slope of the voltage/tension curve) was steeper in patients with muscular dystrophy than in healthy subjects. It was also shown that the latency of the jaw-jerk reflex was longer and the masticatory performance was considerably less in muscular dystrophy than in a healthy subject. However, there were no differences between those with and without an open-bite, which is characteristic of Duchenne type of muscular dystrophy, for all of the tests. From these findings, the authors suggested that the masticatory disturbance in patients with muscular dystrophy is due more to neuromuscular disturbances in the masticatory system rather than to malocclusion such as an open-bite. It was therefore considered that the masticatory disturbance in muscular dystrophy could not be improved by simply increasing the contact points between upper and lower teeth.

Therapeutic exercise has been recommended for

Address for reprint requests: Dr Y Kawazoe, Department of Prosthetic Dentistry, Hiroshima University School of Dentistry, Kasumi 1-2-3, Minami-Ku, Hiroshima 734, Japan.

Received 12 February 1981 and in revised form 6 November 1981

Accepted 6 August 1981 patients with various functional disorders of the muscles of extremities. Unfortunately it is widely believed that it is futile to attempt rehabilitation procedures in patients with muscular dystrophy because muscular degeneration is progressive. Many clinicians, nevertheless, have evaluated the effect of exercise in patients with muscular dystrophy. Abramson and Rogoff ${ }^{2}$ treated 27 patients for a period of 7 months and found improvement in muscle strength, reduction of contractures, and increase in performance of the activities of daily living. Vignos and Watkins ${ }^{3}$ made patients with muscular dystrophy exercise for 1 year and reported that improvement in muscle strength occurred in all patients during the first 4 months of exercise regardless of the type of dystrophy. As mentioned by Rusk, ${ }^{4}$ it is likely that an appropriate physical and occupational therapy programme will prevent disuse atrophy and keep muscular power at a maximal level. Therefore, in order to improve mastication in patients with muscular dystrophy, exercise of the stomatognathic system may be indicated. As far as we know, however, there have been no reports of improvement of masticatory disturbance in muscular dystrophy following exercise. This study evaluated the effects of therapeutic exercise on masticatory function in such patients.

\section{Materials and methods}

\section{Patients}

Seven male patients with Duchenne type of muscular dystrophy, 16 to 24 years of age, were selected. The diagnosis was made after careful clinical study (the pattern 
of muscle weakness, family history, age of onset, and the presence of pseudohypertrophy), serum creatine phosphokinase measurement, electromyography, and muscle biopsy. All the patients had natural dentition and showed open-bite. They could sit on a wheelchair with back support and needed assistance in bed and for wheelchair activities. In this experiment, patients with permanent dentition were selected to avoid variation due to mixed dentition; consequently the subjects were rather older than those with typical Duchenne dystrophy.

\section{EMG recording}

The patients were seated in a wheelchair with the head positioned so that the Frankfort plane was parallel to the floor. Beckman miniature size surface electrodes were affixed to double-sided adhesive tapes placed $20 \mathrm{~mm}$ apart on the skin surface in line with the general direction of the fibres of the masseter muscle on the preferred chewing side. The centre between the electrodes was placed on the centre of the superficial part of the masseter muscle at the same height as the occlusal plane. The resistance between the electrodes was less than $20 \mathrm{k} \Omega$, and an ear clip electrode served as ground.

The electrodes were connected to a preamplifier with an input impedance of $5 \mathrm{M} \Omega$ and with three $\mathrm{dB}$ points on the frequency responses of $16 \mathrm{~Hz}$ and $1 \mathrm{kHz}$, and then the amplified signals were recorded by an electromagnetic oscillograph or averaged by a digital computer (ATAC450, Nihon Koden Kogyo Co., Ltd) after A/D conversion.

\section{Relation between integrated electromygraphic activity of masseter muscle and biting force during rapid isometric contraction}

The amplified signals were rectified and integrated in a

Table 1 Suggested therapeutic exercise of the stomatognathic system

\footnotetext{
Exercise I

Patients clench the jaws as hard as possible after muscle vibration for $5 \mathrm{~min}$ and at $110 \mathrm{~Hz}$, applied to the centre of both masseter muscles while maintaining a medium level of jaw contraction. To be repeated five times within $5 \mathrm{~min}$ after muscle vibration.

Exercise 2

With flexion and extension of the neck, patients open widely and clench the jaw, respectively. The head is then rotated and the jaw moved to the contralateral side. To be repeated five times.

Exercise 3

With flexion and extension of the neck, the tongue is depressed and then elevated. The head is then rotated laterally and the tongue is moved to the contralateral side. To be repeated five times.
}

conventional manner. Direct and integrated EMGs were recorded by the electromagnetic oscillograph having a paper speed of $10 \mathrm{~cm} / \mathrm{s}$. The biting force was obtained from a strain-gauge force transducer (Kawazoe $e t a^{56}$ ) and was recorded by the electromyographic oscillograph simultaneously with EMG recording. A metronome was used to control and to standardise the rate of isometric contraction which was made against the force transducer as hard and as quickly as possible at the rate of 1 clench in $2 \mathrm{~s}$.
The slope of the curve relating integrated electromyographic activity to biting force during rapid isometric contraction (the slope of the voltage/tension curve) is influenced by the position of the transducer. ${ }^{5}$ When the transducer is placed anteriorly on the occlusal plane, the biting force is lower than when it is placed posteriorly if the elevator muscles are excited equally. The slope is also affected by the direction of the force transducer on the occlusal surface of the teeth. Therefore, the bite table was constructed for each patient on the preferred chewing side so that the force transducer might be in the same position and same direction in each recording. The bite table could also standardise the horizontal position of the jaw during clenching. Moreover, the biting force was changed into moment which was obtained by multiplying the biting force by the distance between the transducer and the condylar fulcrum.

The portion in which the biting force increased linearly and as quickly as possible was selected on the recording paper as shown with solid lines (arrows) in fig 1. Then 10 values of the integrated electromyographic activities were plotted against 10 values of the corresponding biting moment (equally spaced in time) within the portion. Since the curve thus obtained showed a linear relationship, the regression equation

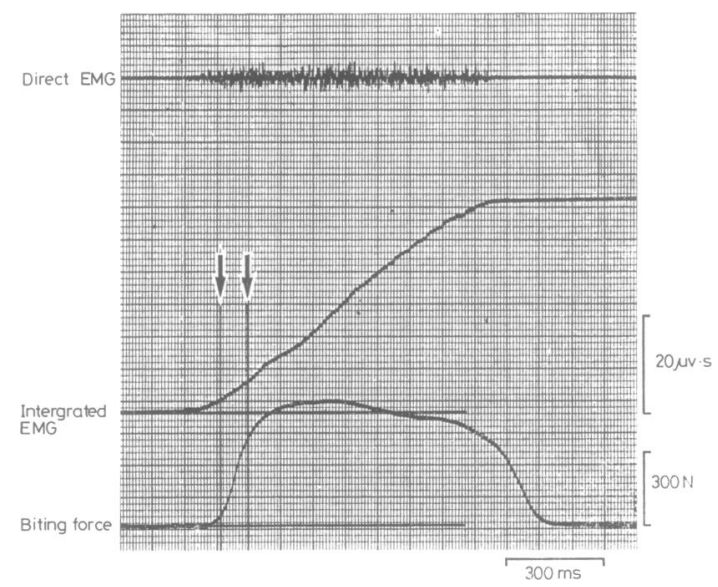

Fig 1 Relation between integrated EMG activity of masseter-biting force during rapid isometric contractions. The portion in which the biting force increased approximately linearly and as quickly as possible was selected for measurement (arrows).

$$
\left(\mathrm{Y}=\mathrm{a}+\mathrm{bX}, \mathrm{b}=\frac{\Sigma \mathrm{xy}-\mathrm{n} \overline{\mathrm{x}} \bar{y}}{\Sigma \mathrm{x}^{2}-\mathrm{n}^{2}}\right)
$$

for each curve was calculated by the least square method and slope (b) was examined in patients with muscular dystrophy before, and 1, 2, 4, and 6 months after the commencement of the therapeutic exercise of the stomatognathic system.

In order to avoid muscle fatigue during measurement three recordings were made for each measurement and average of three slopes obtained from each recording 
showed the slope of each state in each patient. For details concerning the techniques employed, the reader is referred to our previous studies. ${ }^{56}$

\section{Latency of the jaw-jerk reflex}

The patients were instructed to open slightly their jaws and the metal plate was placed on mandibular incisors, which were pressed by the metal plate in the direction of the long axis of the teeth. Then the jaw-jerk reflex was elicited by tapping the metal plate with a hammer (fig 2). The frequency of stimulation for tapping was once a second and the taps were always given in groups of 32 . Sounds elicited by tapping the metal plate were detected by a microphone attached to the zygoma. These sounds triggered the computer and 32 responses after tapping the metal plate were averaged. The latency was measured as the time from detection of tapping sound to the onset of the jaw-jerk reflex. These measurements were also made before and during the therapeutic exercise in the stomatognathic system.

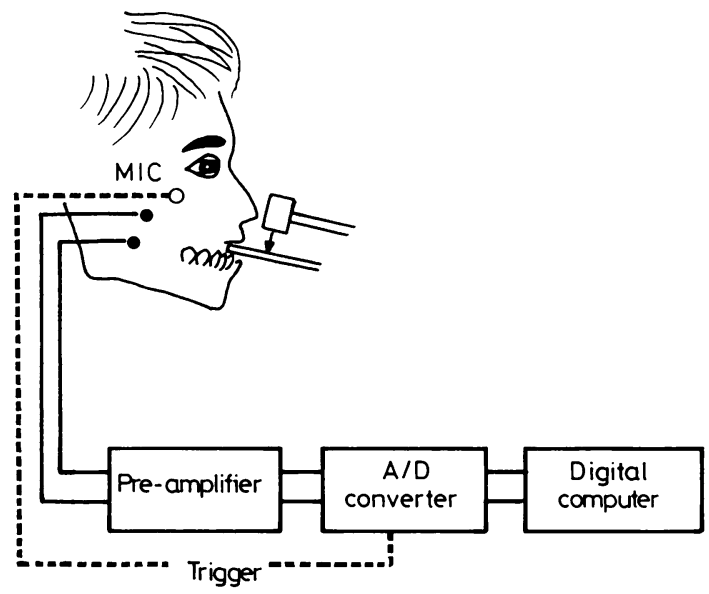

Fig 2 Schematic drawing of experimental apparatus for measurement of the latency of the jaw-jerk reflex.

\section{Masticatory performance}

In order to evaluate the effectiveness of chewing food, measurement of masticatory performance was carried out in accordance with the method devised by Manly and Bradley. ${ }^{7}$ A $15 \mathrm{~g}$ sample of peanuts was divided into five $3 \mathrm{~g}$ portions. The patients were instructed to masticate each portion for 20 masticatory strokes on the preferred chewing side and to avoid swallowing any of the food. The chewed food was spit out into a beaker and the patients were also instructed to rinse their mouth and to add the rinsings to the beaker. The contents of the beaker were stirred gently and poured through 10 mesh screen. Fine particles were washed through the sieve with a jet of water from a wash bottle, and the filtered and passed portions were transferred by washing with water to filter papers in separate funnels. The filters were dried in an oven at $100^{\circ} \mathrm{C}$ for 3 hours, transferred to a desiccator for 2 hours, and weighed. Performance was calculated as being the volume of particles passing the 10 mesh screen divided by total volume of peanuts collected from the mouth. These measurements were performed before and during therapeutic exercise.

\section{Therapeutic exercise of the stomatognathic system}

The exercise programme used in this experiment is shown in table 1. Various facilitation techniques have been used during therapeutic exercise to improve voluntary movement. In recent years, the tonic vibration reflex has been so used. Watanabe ${ }^{8}$ has reported that patients with paralysed limb muscles can learn a pattern of exercise during post-vibratory potentiation, and suggested that this can be used as a facilitation technique. It is known that the post-vibratory potentiation is induced effectively by vibration longer than $5 \mathrm{~min}$, and at high frequency $(110 \mathrm{~Hz})$ with the subject postured so that muscle tone was increased. ${ }^{9}$ Moreover, this potentiation occurs even in masseter muscle in patients with muscular dystrophy and continues for $5 \mathrm{~min}$ after vibration. ${ }^{10}$ Accordingly, we recommended muscle vibration at $110 \mathrm{~Hz}$ for $5 \mathrm{~min}$ applied to the centre of both masseter muscles with the patient clenching the jaw at medium level, and intermittent clenching was performed during the postvibratory potentiation (Exercise 1).

The tonic neck reflex has also been used as a facilitation technique. Funakoshi et $a l^{11}$ has observed that voluntary alteration of head posture alters contraction of the jaw musculature; EMGs of the jaw muscles, especially the digastric muscles, increased in response to neck flexion, while during extension of the neck the temporal muscles were most active. When the head was rotated to the left, the left temporal, masseter, and digastric muscles were activated much more than their contralateral counterparts; reciprocal responses were seen when the head was rotated in the opposite direction. Therefore, in order to facilitate mandibular movements during mastication, tonic neck reflexes were used (Exercise 2).

Tongue movements are also reinforced by the position of the head $;^{12}$ flexion and extension of the neck reinforces depression and elevation of the tongue, respectively; rotation of the head also reinforces lateral movements of the tongue. On the basis of this finding, alteration of the head posture was used to facilitate tongue movements (Exercise 3). These exercises were supervised by a physiotherapist for each patient once a day. As the patients could exercise the jaw isometrically (intermittent clenching) by themselves, they were instructed to carry out the isometric exercise many times a day.

\section{Results and Discussion}

\section{EFFECTS OF THERAPEUTIC EXERCISE ON THE SLOPE OF THE VOLTAGE/TENSION CURVE}

Mean slope of the voltage/tension curves obtained from seven patients was slightly decreased during the exercise and reached constant level at 2 months of the exercise (table 2, fig 3). Means of the slopes before and after 6 months of exercise were $1 \cdot 10$ and $0 \cdot 85$, respectively, but there was no difference between them ( $\mathrm{p}<0 \cdot 1, t$ test). 
Table 2 Effects of the therapeutic exercise in the stomatognathic system

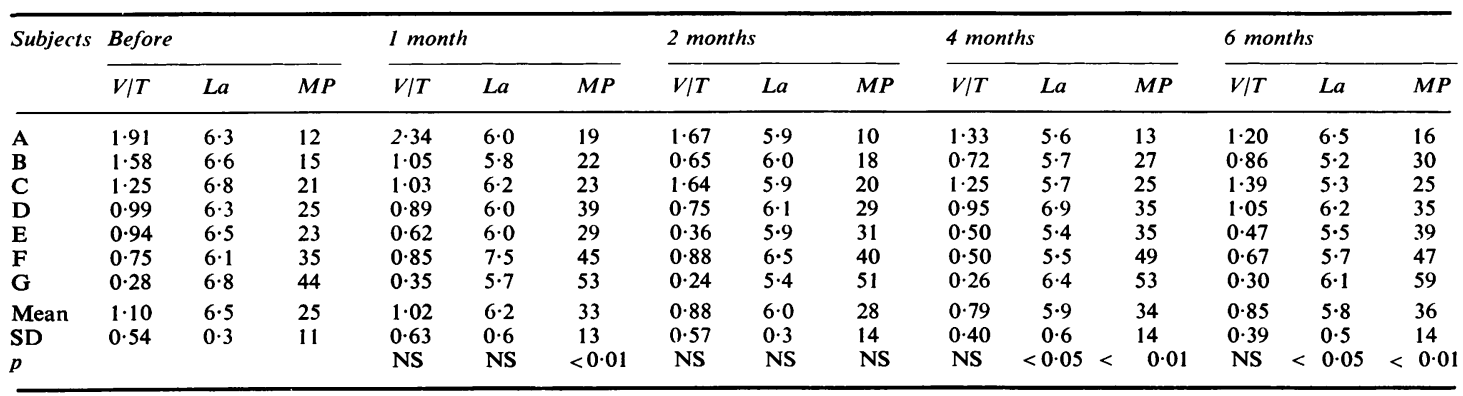

$\mathrm{V} / \mathrm{T}$-slope of the voltage/tension curve; La-latency of the jaw-jerk reflex in $\mathrm{m}$; MP-masticatory performance in percentage. NS-not significant.

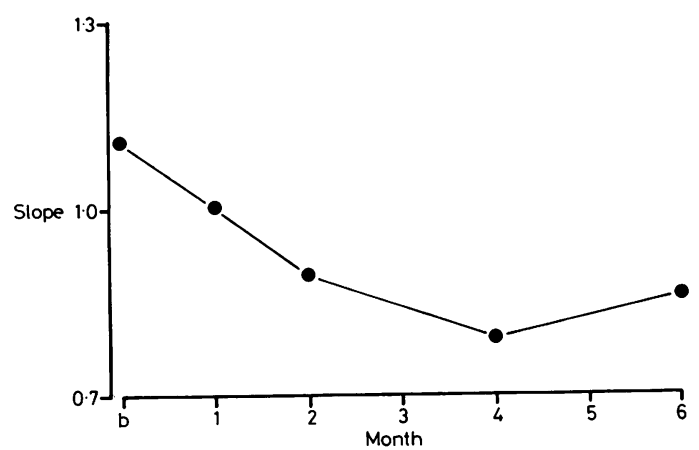

Fig 3 Effect of exercise on the slope of the voltage/ tension curve. Ordinate: the slope of the voltage/tension curve in $\mu \mathrm{Vs} / \mathrm{Nm}$. Abscissa: duration of therapeutic exercise in months. $b$ : before exercise.

The slope of the curve relating integrated electromyographic activity to biting force may be regarded as a measure of the efficiency of the individual fibres composing a muscle. ${ }^{5}$ The more ineffective the muscle fibres as contractile units, the more fibres will be required to exert tension and the steeper will be the voltage/tension curve.

It is known that the blood flow in muscle is improved ${ }^{1314}$ and mitochondrial respiratory enzyme activity in the skeletal muscle is increased ${ }^{15}$ by muscle training, so that the efficiency of energy utilisation is enhanced by the training. ${ }^{16}$ Consequently, it was predicted that the slope of the voltage/tension curve, which indicates the efficiency of the muscle fibres, would be decreased by exercise in this experiment but it did not. However, necrosis and myophagia of muscle fibres, and infiltration by fatty tissue into the stroma of the skeletal muscle in the Duchenne type of muscular dystrophy have been observed. ${ }^{17}$ It is presumably owing to this change in the muscle fibres that there was no dif- ference between the slopes of voltage/tension curves before and after exercise.

EFFECT OF THERAPEUTIC EXERCISE ON THE LATENCY OF THE JAW-JERK REFLEX

Table 2 and fig 4 show the result obtained from the measurements before and during the therapeutic exercise. Mean latency of the jaw-jerk reflex obtained from seven patients was also decreased during the exercise and reached a constant level at 2 months of the exercise. However, there was no difference

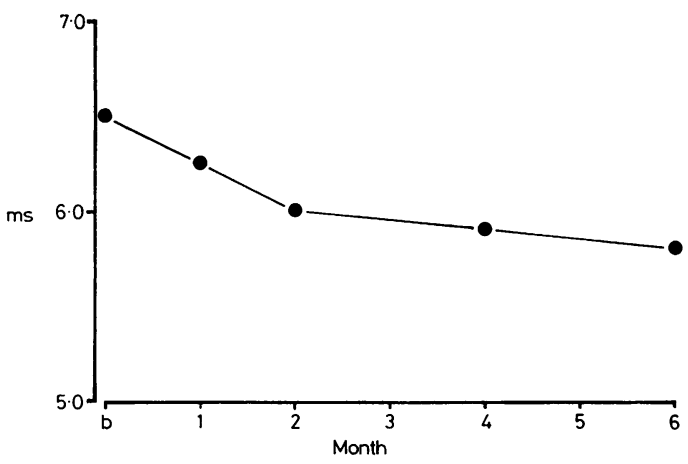

Fig 4 Effect of exercise on the latency of the jaw-jerk reflex. Ordinate: the latency of the jaw-jerk reflex in $\mathrm{ms}$. Abscissa: duration of therapeutic exercise in months. $b$ : before exercise.

between mean values before and 6 months of the exercise $(\mathrm{p}<0 \cdot 1, t$ test $)$.

Eccles and McIntyre ${ }^{18}$ have investigated the effect of inactivity of monosynaptic spinal reflex arcs in the cat. They recorded the reflex responses evoked in different hindlimb muscle nerves by stimulation of dorsal roots severed several weeks previously, using responses of the normal reflex arcs of the opposite side as controls. The latent period of the 
monosynaptic reflex was considerably longer $(0.6 \mathrm{~ms})$ on the operated side. On the other hand, Eccles and Westerman ${ }^{19}$ reported that excess activity led to enhancement of synaptic efficacy. Kozac and Wester$\operatorname{man}^{20}$ also reported that increased synaptic use led to shortening of the latent period, and to an increase in the amplitude of the monosynaptic reflex.

On the basis of these findings, the longer reflex latency in muscular dystrophy may result in part from disuse of the reflex arc caused by muscular weakness. The latency of the jaw-jerk was decreased by exercise (fig 4).

\section{EFFECT OF THERAPEUTIC EXERCISE ON}

MASTICATORY PERFORMANCE

Masticatory performance was increased during the period of therapeutic exercise (table 2, fig 5), and the significant changes were observed at 1, 4, and 6 months of the exercise (table 2). Consequently, subjective improvements in masticatory function were occurred in six out of seven. Most of patients reported that it was easier to masticate and two patierits reported that it was easier to chew meat.

From these findings, therapeutic exercise of the stomatognathic system is effective in improving masticatory function in patients with progressive muscular dystrophy.

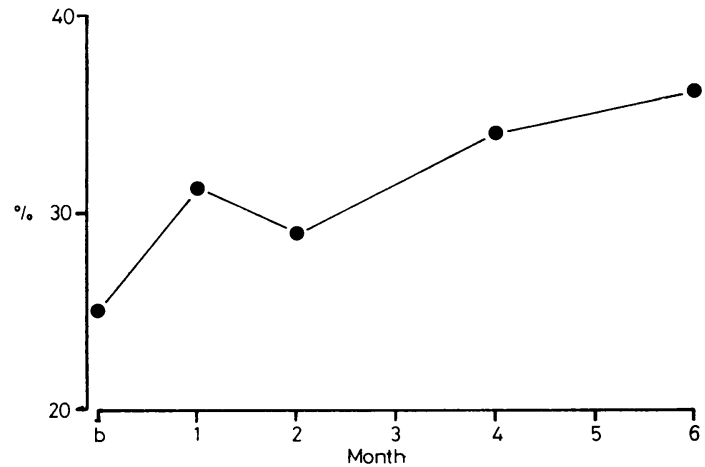

Fig 5 Effect of exercise on masticatory performance. Ordinate: masticatory performance (measure as described in Methods). Abscissa: duration of therapeutic exercise in months. b: before exercise.

\section{References}

${ }^{1}$ Kawazoe Y, Kotani H, Kobayashi M, Hamada T. Electromyographic study of masticatory muscles in progressive muscular dystrophic patients. J Dent Res 1978;57 (Special Issue A):914.

${ }^{2}$ Abramson AS, Rogoff J. Physical treatment in muscular dystrophy. Proc Second Medical Conference of Muscular Dystrophy Assoc of America 1952;123-4.

${ }^{3}$ Vignos PJ, Watkins MP. The effect of exercise in muscular dystrophy. $J A M A$ 1966;197:843-8.

${ }^{4}$ Rusk HA. Rehabilitation Medicine. Saint Louis: CV Mosby Co, 1977:447.

${ }^{5}$ Kawazoe Y, Kotani H, Hamada T. Relation between integrated electromyographic activity and biting force during voluntary isometric contraction in human masticatory muscles. J Dent Res 1979;58:1440-9.

${ }^{6}$ Kawazoe Y, Kotani H, Maetani T, Yatani H, Hamada $T$. Integrated electromyographic activity and biting force during rapid isometric contraction of fatigued masseter muscle in man. Archs Oral Biol (in press).

7 Manly RS, Bradley LC. Masticatory performance and efficiency. J Dent Res 1950;29:448-62.

${ }^{8}$ Watanabe S. Tonic vibration reflex. Clin Electroencephalogr $1971 ; 13: 449-57$ (in Japanese).

9 Yamanaka T. Effect of high frequency vibration on muscle spindle in the human body. $J$ Chiba Med Soc 1964;40:338-46 (in Japanese).

${ }^{10}$ Kawazoe Y, Kobayashi M. Unpublished observation.

${ }^{11}$ Funakoshi M, Fujita N, Takehana S. Relations between occlusal interference and jaw muscle activities in response to change in head position. J Dent Res 1976;55:684-90.

${ }^{12}$ Knott M, Voss DE. Proprioceptive Neuromuscular Facilitation. Pattern and Technique. New York: Hoeber-Harper Book, 1956:107.

13 Vanderhoof ER, Imig CJ, Hines HM. Effect of muscle strength and endurance development on blood flow. J Appl Physiol 1961;16:873-7.

${ }^{14}$ Rohter FD, Rochelle RH, Hyman C. Exercise blood flow changes in the human forearm during physical training. J Appl Physiol 1963;18:789-93.

${ }^{15}$ Holloszy JO. Effects of exercise on mitochondrial oxygen uptake and respiratory enzyme activity in skeletal muscle. $J$ Biol Chem 1967;242:2278-82.

${ }^{16}$ Karpovich PV. Physiology of Muscular Activity. Philadelphia: WB Saunders Co, 1965:107.

17 Daniel PM, Strich SJ. Skeletal muscle. In: Symmers WC, ed. Systemic Pathology. Edinburgh: Churchill Livingstone, 1979:2350.

${ }^{18}$ Eccles JC, McIntyre AK. The effect of disuse and of activity on mammalian spinal reflexes. $J$ Physiology (Lond) $1953 ; 121: 492-516$.

${ }^{19}$ Eccles RM, Westerman RA. Enhanced synaptic function due to excess use. Nature 1959;184:460-1.

${ }^{20}$ Kozac W, Westerman RA. Plastic changes of spinal monosynaptic responses from tenotomized muscle in cats. Nature $1961 ; 189: 753-5$. 